\title{
Quasi-free one-nucleon Knockout Reactions on neutron-rich Oxygen Isotopes
}

\section{Leyla Atar ${ }^{*}, 1$, , Stefanos Paschalis ${ }^{2}$, Thomas Aumann ${ }^{1}$, Carlos Bertulani ${ }^{3}$ for the $\mathbf{R}^{3} \mathbf{B}$-collaboration}

${ }^{1}$ Institut für Kernphysik, Technische Universität Darmstadt, D-64289 Darmstadt, Germany

${ }^{2}$ Department of Physics, University of York, York YO10 5DD, United Kingdom

${ }^{3}$ Department of Physics \& Astronomy, Texas A\&M University Commerce, Commerce, TX 75429, USA

According to the Independent Particle Model (IPM) the single particle states are fully occupied with a spectroscopic factor one. However in electron-induced proton knockout reactions a reduction of single-particle strengths has been observed to about $60-70 \%$ for stable nuclei in comparison to the IPM. This finding has confirmed by nuclear knockout reactions using stable and exotic beams, however, with a strong dependency on the proton-neutron asymmetry. To understand this dependency quantitatively a complementary approach, quasi-free reactions, is introduced. Quasi-free knockout reactions in inverse kinematics at relativistic energies allows us to investigate singleparticle structure of stable and exotic nuclei in a most direct way.

We have performed a systematic study of spectroscopic strength of oxygen isotopes using quasi-free $(p, 2 p)$ and (p,pn) knockout reactions in complete kinematic at the $\mathrm{R}^{3} \mathrm{~B} / \mathrm{LAND}$ setup (at GSI in Darmstadt, Germany) with secondary beams containing ${ }^{13-24} \mathrm{O}$. The oxygen isotopic chain offers a large variation of separation energies, which allow us to obtain a quantitative understanding of spectroscopic factors with respect to isospin asymmetry.

The ${ }^{16-18} \mathrm{O}$ and ${ }^{21-23} \mathrm{O}$ isotopes have been analyzed so far and the preliminary results will be presented. The results include cross sections, momentum distributions and gamma-coincidence measurements. By comparison with the eikonal reaction theory the spectroscopic and reduction factors have been extracted and will be compared to existing data.

54th International Winter Meeting on Nuclear Physics

25-29 January 2016

Bormio, Italy

* Speaker

§Email: l.atar@gsi.de 


\section{Introduction}

The main interest behind the presented work is the study of single-particle structure and its evolution with respect to proton-neutron asymmetry towards proton and neutron driplines. The Independent Particle Model (IPM) describes nucleons as single-particle moving independently in a mean-field potential created by all nucleons. In this model the single-particle states below the Fermi level are fully occupied with spectroscopic factors of unity. The model is applicable only to nuclei with a single particle or hole state on the closed shell and explains the very basic properties of nuclei very well. Nevertheless, IPM is an approximation and deviations from this picture arise from residual interactions, which is not included in IPM. The model IPM builds the main concept of the Shell Model (SM) which includes residual interactions and describes many properties of nuclei successfully.

The electron-induced proton-knockout reactions at the NIKHEF facility [1,2] have shown a deviation from the description of the IPM. The quantity $R$, the so-called reduction factor, demonstrates this deviation and is defined as the ratio of the experimental cross section to the theoretical prediction relative to IPM. These studies show a reduction of single-particle strength of about $30-40 \%$ in comparison to IPM for different target masses. This reduction is due to the partially occupation of single-particle states and a common interpretation of the origin of this is correlation effects, which is not included in IPM. These correlations are such as short-range, long-range correlations and tensor interaction [3-6]. A consequence of these correlations is the configuration mixing of single-particle states near the Fermi surface or scattering of nucleons to high momenta states.

However, the measurements at NIKHEF facility were restricted to proton valence states and stable isotopes. With the availability of exotic beams, the measurements are expanded to large isospin asymmetric systems using one-nucleon removal reactions at intermediate energies, it is also called knockout reactions. A recent compilation of the existing data from hadron and electron induced knockout reactions were reported by J.A. Tostevin and A. Gade [7]. These results show a strong isospin dependency of the single-particle strength, namely the strongly bound nucleons reveal a larger reduction then loosely bound nucleons. In contrast, the results from transfer reactions suggest a reduction of the strength but do not indicate such strong dependency [8]. On the other hand, ab-initio calculations, such as self-consistent Green's functions method based on the chiral three-nucleon forces (3NFs) at NNLO [9], or coupledcluster calculations using the $\mathrm{N}^{3} \mathrm{LO}$ interaction model [10] have predicted the effect of an asymmetry dependence of the single-particle strength similar to that observed experimentally by the knockout reactions but considerably weaker in magnitude.

This isospin dependence is still an ongoing discussion and the origin is unclear whether it is a indication of higher order correlations missing in the SM calculations or an artifact of the reaction model. Because knockout reactions on composed targets, such as carbon or beryllium, at intermediate energies around 50-100 MeV/u are mostly peripheral collisions [11]. Due to the strong absorption in the nuclear medium, knockout reactions are concentrated at the surface. 
That allows to probe the outer part of the projectile wave function and constrains the sensitivity to higher-lying states. To validate these findings, a complimentary approach, quasi-free scattering (QFS) reactions, is introduced in the next sections.

\subsection{Quasi-free scattering reactions}

The QFS reactions in inverse kinematics using proton target at high beam energies (0.2-1 $\mathrm{GeV} / \mathrm{u}$ ) offers an alternative approach to investigate the single-particle strength for a wide range of nuclei from the valley of stability towards driplines.

Proton targets enable a quasi-free nucleon-nucleon reaction and increase the sensitivity to deeply bound states. It allows a complete investigation of the projectile wave function, giving access to different regions of a nucleus [12].

At high beam energies the absorption in the nuclear medium is weaker due to minimized rescattering in the final state, because the nucleon-nucleon interaction cross section reaches its minimum around 300 MeV [13]. At these energies, the de-Broglie wave length of the projectile is small with respect to the nuclear distances which supports the picture of a localized reaction. The reaction mechanism is based on the eikonal model [12] with the assumption that the reaction happens between the knocked-out nucleon in the nucleus and the target proton. In a QFS reaction, the knocked-out nucleon and the target proton are scattered with an opening angle around $90^{\circ}$ and both nucleons are detected [14]. The residual nucleus receives a recoil momentum which corresponds to the internal momentum of the knocked-out nucleon but in opposite direction.

\subsection{Oxygen isotopic chain}

The current work addresses the experiment of quasi-free knockout reactions on the oxygen isotopic chain. Because it offers a large variation of separation energies, as can be seen in Fig.1. The difference in proton and neutron separation energies $\Delta S$ is shown as a function of neutron excess. $\Delta S$ reflects the isospin asymmetry and the positive value of $\Delta S$ indicates strongly bound nucleons while negative value of $\Delta S$ shows loosely bound nucleons. The blue stars in Fig.1 show the proton separation energy minus neutron separation energy indicating proton-knockout reactions and the red stars vice versa representing neutron-knockout reactions [15]. It spans a sizeble range and hence allows to obtain a quantitative understanding of reduction factors in a large variation of isospin asymmetry. In the current experiment, the projectiles from the proton to neutron driplines are available for a systematic analysis of (p,2p) and (p,pn) reactions.

\section{2. $\mathbf{R}^{3} \mathbf{B} / \mathrm{LAND}$ experimental setup at GSI}

The present experiment was performed at the $\mathrm{R}^{3} \mathrm{~B} / \mathrm{LAND}$ experimental setup at $\mathrm{GSI}^{1}$ in Darmstadt, Germany. The primary argon beam was first accelerated at the linear accelerator UNILAC $^{2}$ and then at the ring accelerator SIS18 ${ }^{3}$ [16] up to the energy $500 \mathrm{MeV} /$ nucleon and

\footnotetext{
${ }^{1}$ Gesellschaft für SchwerIonenforschung

${ }^{2}$ UNIversal Linear Accelerator

${ }^{3}$ SchwerionenSynchrotron, Heavy Ion Synchrotron
} 
then impinged on the Be target for the fragmentation. The fragments are selected using the inflight technique at the fragment separator (FRS) [17] and then delivered to the experimental hall Cave C.

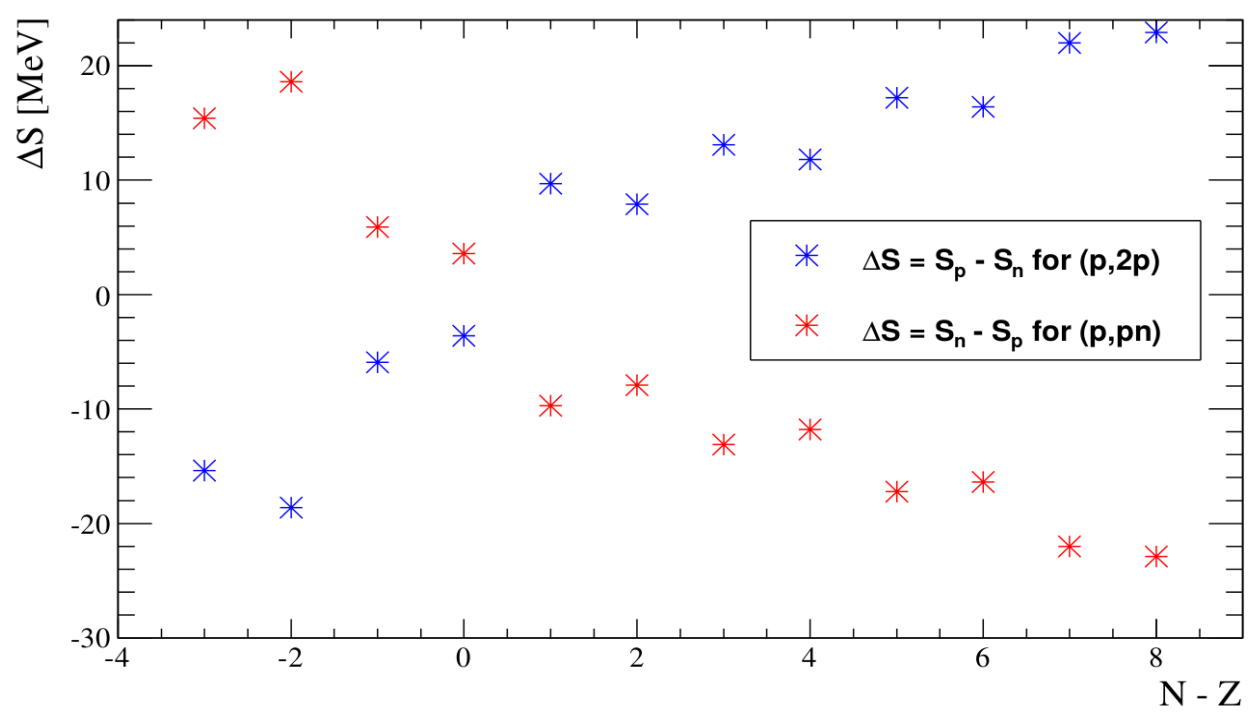

Figure 1: Illustration of proton-neutron asymmetry for the oxygen isotopic chain. Difference in proton and neutron separation energies $\Delta \mathrm{S}$ are plotted against neutron excess.

The experimental setup shown in Fig2. provides kinematically complete measurements, i.e. all reaction products including gamma-rays are detected, of heavy ions and light nucleons in inverse kinematics. Inverse kinematics means that the nuclei of interest are fast projectiles produced in fragmentation reactions.

The secondary beams, containing ${ }^{13-24} \mathrm{O}$ at energies around $400-500 \mathrm{MeV} / \mathrm{u}$, were identified using the plastic scintillator $\mathrm{POS}^{4}$ for the time measurements and the silicon detector PSP $^{5}$ for the energy-loss measurements. Combining these informations with the time measured in the plastic scintillators at the focal plane S8 of the FRS, the incoming nuclei can be identified using the mass-to-charge ratio relation $\mathrm{B} \rho=\beta \mathrm{V} / \mathrm{Z}$.

The production target is located inside the Crystal Ball (CB) calorimeter and surrounded by the silicon strip detectors (DSSDs) which provide energy-loss and position measurements and used for the tracking procedure. The CB consists of 162 sodium iodide crystals and detects gammarays from the de-excitation of fragments in almost $4 \pi$ direction. It is also suited to detect nucleons from the knockout reactions in the forward hemisphere. The reaction products pass then through the dipole magnet ALADIN which bends the flight path of fragments and protons, while neutrons are not affected. The heavy fragments are bent towards the fragment arm

\footnotetext{
${ }^{4}$ POSition detector

${ }^{5}$ Position Sensitive silicon Pin diode
} 
(indicated by the red arrow) of the setup, consisting of two fiber detectors GFIs and a time-offlight wall TFW. The green line represents the neutron arm for the detection of neutrons in forward direction to the LAND detector. The proton arm is not depicted in the figure for simplicity.

The incoming beam and reaction products were identified from the energy-loss and time-offlight as well as the position measurements in various detectors in an event-by-event basis. The measurements were performed using $\mathrm{CH}_{2}$, carbon and empty-run targets.

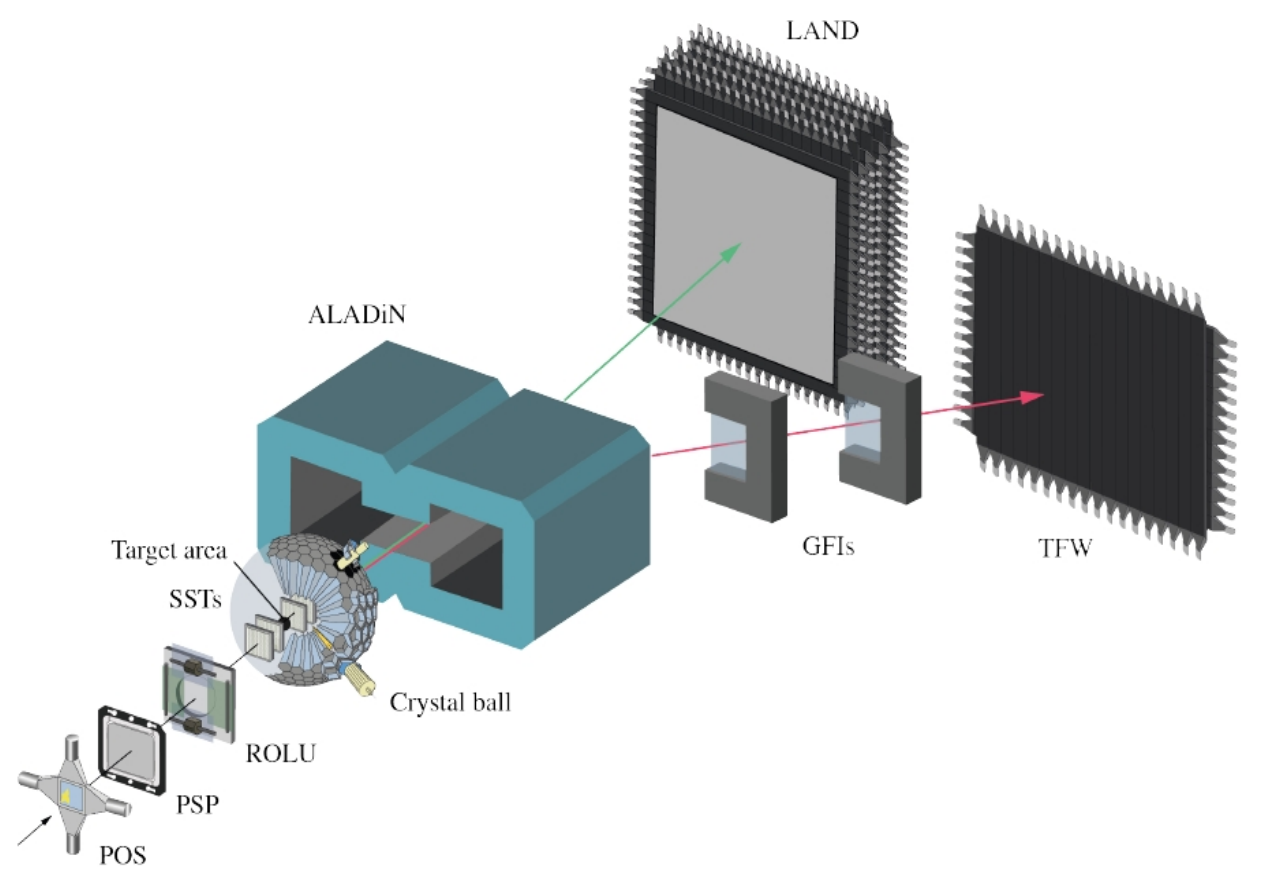

Figure 2: Schematic layout of the $\mathrm{R}^{3} \mathrm{~B} / \mathrm{LAND}$ setup in the experimental area Cave $\mathrm{C}$ used in the discussed experiment. Picture taken from [18].

\section{Experimental Results}

As explained in the previous section, the incoming cocktail beam was identified by means of velocity, energy-loss and magnetic rigidity analysis. The correlation of reconstructed charge and mass-to-charge ratio allows to identify unambiguosly the incoming ions, as depicted Fig.3.

The charge identification of the fragments after the collision was done via energy-loss measurements in DSSDs located directly behind the target in the beam line and in TFW at the end of the fragment arm.

The masses of the fragments after collisions were reconstructed by using a dedicated tracking procedure based on position measurements from the DSSDs in front of the magnet and two fiber detectors GFIs behind it together with the time-of-flight measured by TFW. Fig.4 shows the mass distribution of fragments from the projectile ${ }^{16} \mathrm{O}$ on the $\mathrm{CH}_{2}$ target measured in 
coincidence with a proton in CB. The left histogram (a) shows the fragment masses with $\mathrm{Z}=8$, whereas the right histogram (b) displays fragment masses with $\mathrm{Z}=7$.

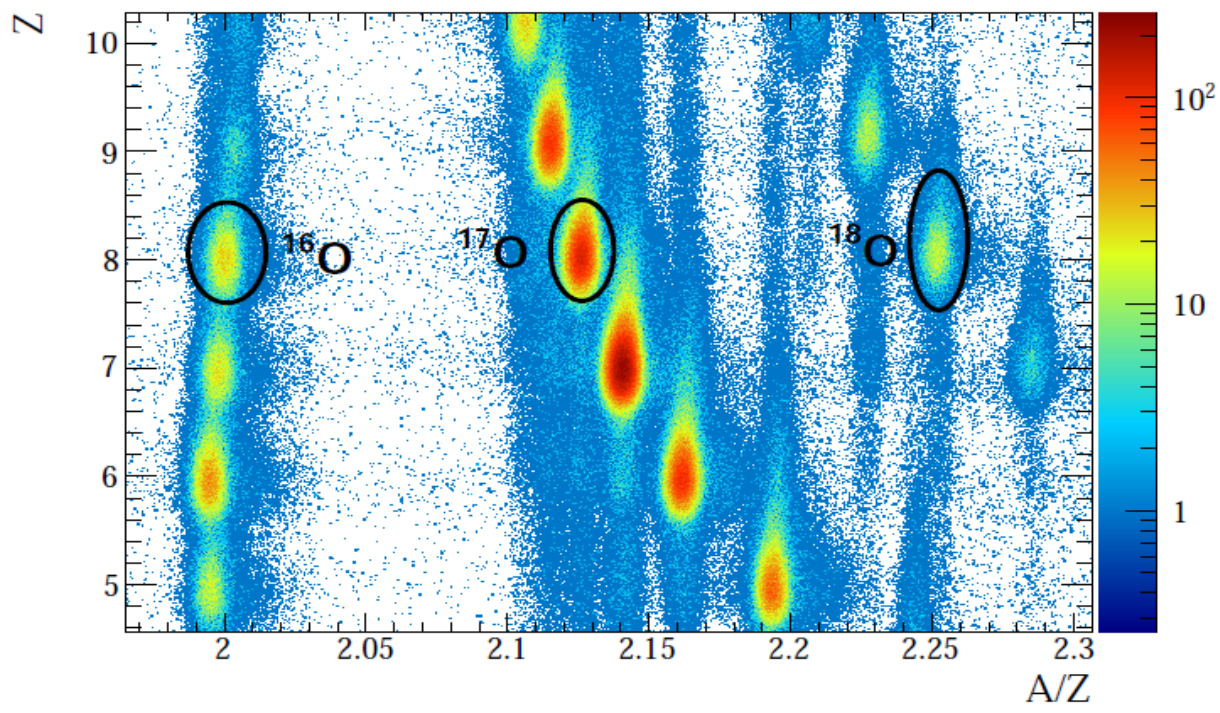

Figure 3: Identification of projectiles of interest ${ }^{16-18} \mathrm{O}$ indicated with the ellipses. The charge of isotopes are plotted against the mass-to-charge ratio.

Coincidence measurements of the knocked-out nucleon and the target proton as well as the residual fragment allow for an unambiguous reconstruction of the quasi-free reaction channels and to obtain the inclusive reaction cross sections. As mentioned before, the measurements were performed using $\mathrm{CH}_{2}$ and carbon as well as empty-run targets. The carbon target is used to estimate carbon induced reactions in the $\mathrm{CH}_{2}$ target while empty-run target is used to estimate the background contribution. The reactions with the proton target imply the interactions with the protons in the $\mathrm{CH}_{2}$ target and was obtained by subtracting the carbon induced as well as background reactions from the measurements on the $\mathrm{CH}_{2}$ target.

The ground state configuration of the projectiles are based on the IPM description. The measurement of the inclusive cross sections are restricted to the ground state and to the bound excited states of the residual fragment. These states lie also below the nucleon decay energy. The analysis of ${ }^{16} \mathrm{O}$ will be presented in details as a benchmark case and the analysis of the other projectiles are similar.

In a quasi-free $(p, 2 p)$ reaction of the projectile ${ }^{16} \mathrm{O}$, one-proton can be knocked-out either from the $0 \mathrm{p}_{1 / 2}$ shell, in this case the residual fragment ${ }^{15} \mathrm{~N}$ is in its ground state, or from the $0 \mathrm{p}_{3 / 2}$ shell leaving the residue in the excited state with the spin and parity $3 / 2^{-}$. For a knockout from the $0 \mathrm{~s}_{1 / 2}$ shell, the residual fragment is unbound and thus is not considered in the analysis. Therefore, the total cross section consists of the contributions from the six valence protons from both shell. The observed gamma-spectrum in Fig.5 shows the populated states in the quasi-free reaction ${ }^{16} \mathrm{O}(\mathrm{p}, 2 \mathrm{p}){ }^{15} \mathrm{~N}$ and thus confirms this assumption. 


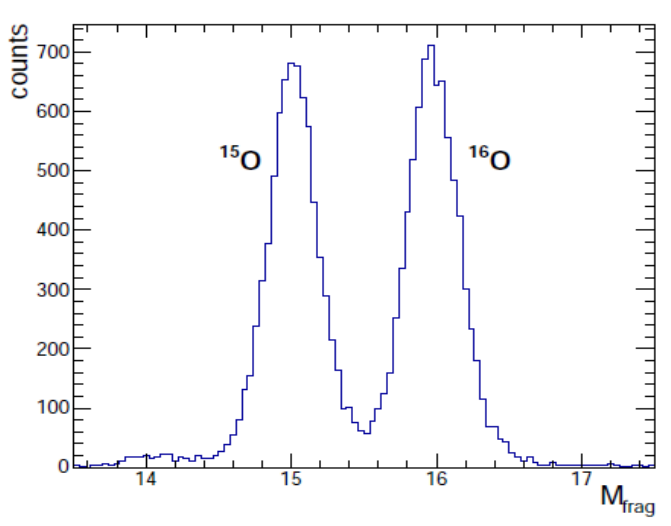

(a) Fragment mass for $\mathrm{Z}=8$

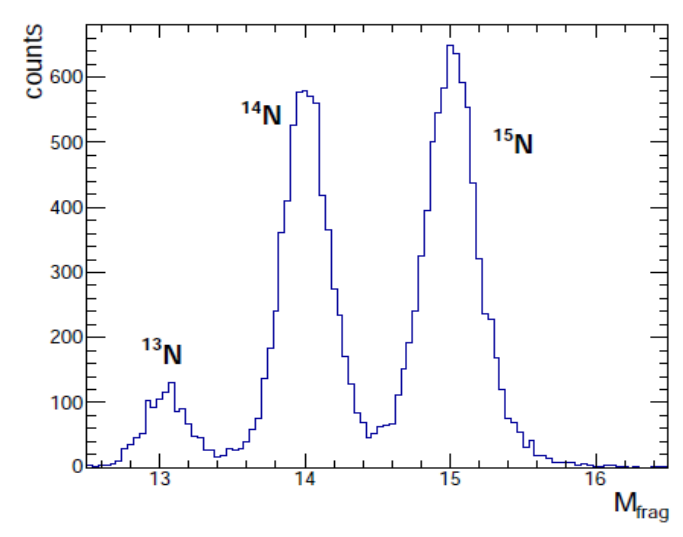

(b) Fragment mass for $\mathrm{Z}=7$

Figure 4: Reconstructed mass distribution of the fragments from the projectile ${ }^{16} \mathrm{O}$ on the $\mathrm{CH}_{2}$ target measured in coincidence with a proton in CB. (a) shows the fragment masses for $Z=8$. (b) shows fragment masses with $\mathrm{Z}=7$.

Fig.5 shows the Doppler-corrected gamma-ray spectrum measured in coincidence with the outgoing fragments and two protons. As can be seen, the strong peak around $6 \mathrm{MeV}$ corresponds to the dominant $3 / 2^{-}$transition to the ground state. There is also a weak peak visible at around 10 $\mathrm{MeV}$ corresponding to the fragmentation of the $3 / 2^{-}$strength at $9.9 \mathrm{MeV}$. The level scheme of ${ }^{15} \mathrm{~N}$ is shown on the right-hand side and the observed transitions are denoted by thick arrows. The population of excited states below the particle decay threshold was determined by fitting simulated detector responses using Geant3 and R3BRoot [19] toolkits to the experimental spectrum. Based on the observed gamma spectrum, the excited 3/2 at $6.32 \mathrm{MeV}$ (red line) and at $9.93 \mathrm{MeV}$ (gray line) as well as the background (green line) were simulated and fitted to experimental data. Utilizing the fit results, the exclusive cross sections of the populated states in the reaction were extracted. Combining of the exclusive cross sections with the theoretical predictions, the spectroscopic factors were deduced.

In the treatment of the QFS reaction, commonly the theoretical DWIA ${ }^{6}$ is used with a complex optical potential [20]. The DWIA calculations are based on the eikonal theory and include the occupation numbers for each considered state according to the IPM with the assumption of a unique spectroscopic factor. In the theory, multiple scatterings are treated using the Glauber model and absorption is taken into account in the complex optical potential [12]. The theoretical calculations [21] were performed for the states based on the observed gamma spectra and the reduction factors obtained from the comparison of the experimental cross section to the calculated one. The obtained reduction factor for the reaction ${ }^{16} \mathrm{O}(\mathrm{p}, 2 \mathrm{p}){ }^{15} \mathrm{~N}$ is consistent with the measurements from the (e,e'p) experiments [22-24]. The shape and width of the theoretical momentum distributions [21] have been compared to the experimental data and show good

\footnotetext{
${ }^{6}$ Distorted Wave Impuls Approximation
} 
agreement. This indicates a confirmation of the proton knockout with the angular momentum $l=1$.

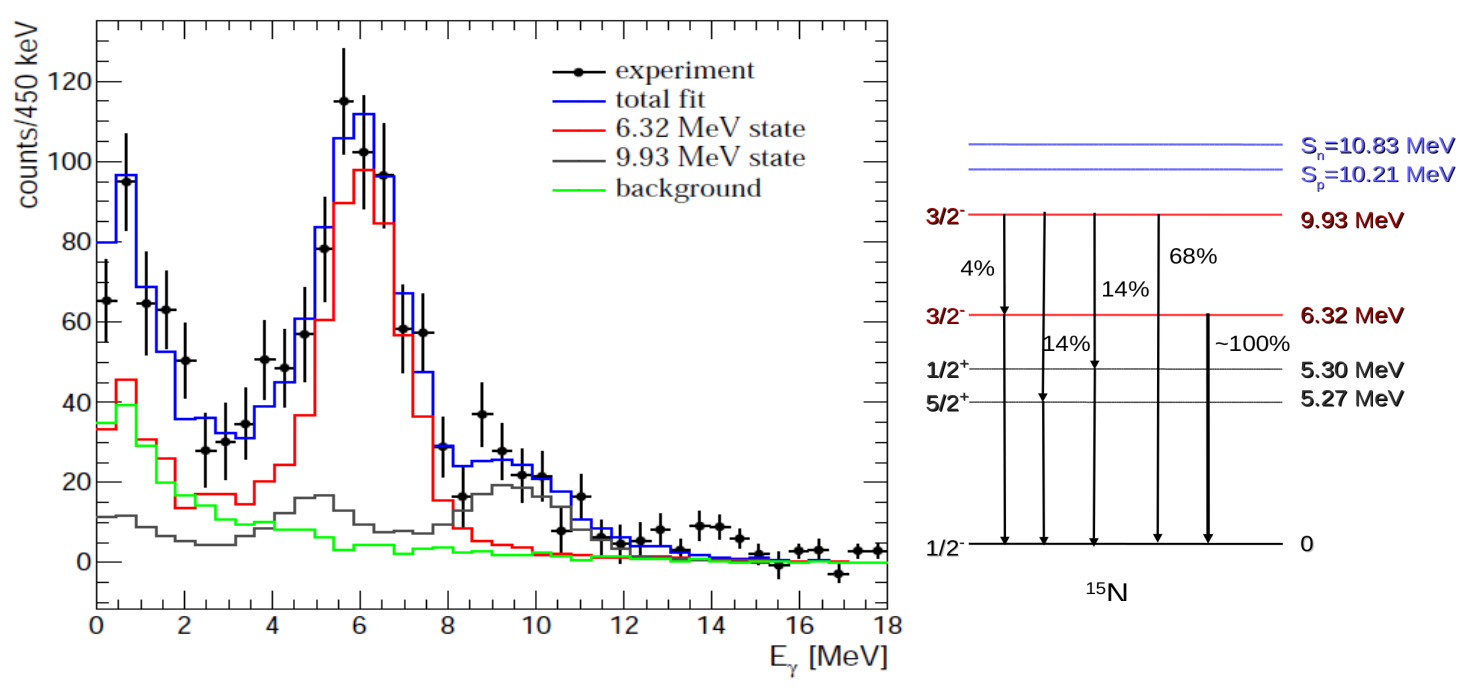

Figure 5: Left: Doppler-corrected gamma spectrum measured in coincidence with ${ }^{15} \mathrm{~N}$ residual fragments on the $\mathrm{H}_{2}$ target. The simulated 3/2- excited state at $6.32 \mathrm{MeV}$ (red line) and at 9.93 $\mathrm{MeV}$ (gray line) as well as the background (green line) were fitted to experimental data, and the blue line indicates the total fit. Right: The level scheme of ${ }^{15} \mathrm{~N}$. The proton and neutron separation energies, $S_{p}$ and $S_{n}$ respectively, are denoted by the blue color while the populated excited states by the red color. The observed transitions are denoted by thick arrows.

A similar analysis for the isotopes in the oxygen chain is currently ongoing. The investigation of both quasi-free proton and neutron knockout will be one of the main part of the research program of the $\mathrm{R}^{3} \mathrm{~B}$-collaboration for the future FAIR project [25]. The concept and the detectors for the future program are being developed and tested at the present $\mathrm{R}^{3} \mathrm{~B}$-LAND setup at GSI.

This work was supported by the German Federal Ministry for Education and Research (BMBF project 05P12RDFN1), by HIC for FAIR, and through the GSI-TU Darmstadt cooperation agreement.

\section{References}

[1] I. Sick, Correlations in Nuclei, PPNP 59(1):447 - 454 (2007).

[2] L. Lapikás, Quasi-Elastic Electron Scattering off Nuclei, Nucl. Phys. A 553, 297c (1993).

[3] V.R. Pandharipande, I. Sick and P. K. A. deWitt Huberts, Independent particle motion and correlations in fermion systems, Rev. Mod. Phys. 69, 981 (1997).

[4] T. Otsuka, Exotic nuclei and nuclear forces, Phys. Scr. T152:014007 (2013). 
[5] D. Rohe, et al., Correlated Strength in the Nuclear Spectral Function, Phys. Rev. Lett. 93, 182501 (2004).

[6] E. Piasetzky, et al., Evidence for Strong Dominance of Proton-Neutron Correlations in Nuclei, Phys. Rev. Lett. 97, 162504 (2006).

[7] J. A. Tostevin and A. Gade, Systematics of intermediate-energy single-nucleon removal cross sections, Phys. Rev. C 90:057602 (2014).

[8] B. P. Kay, J. P. Schiffer, and S. J. Freeman, Quenching of Cross Sections in Nucleon Transfer Reactions, Phys. Rev. Lett. 111, 042502 (2013).

[9] A. Cipollone, C. Barbiere, and P. Navratil, Chiral three-nucleon forces and the evolution of correlations along the oxygen isotopic chain, Phys. Rev. C 92, 014306 (2015).

[10] O. Jensen, G. Hagen et al., Quenching of Spectroscopic Factors for Proton Removal in Oxygen Isotopes, Phys. Rev. Lett. 107, 032501 (2011).

[11] A. Gade and T. Glasmacher, In-beam nuclear spectroscopy of bound states with fast exotic ion beams, PPNP 60:161-224 (2008).

[12] T. Aumann, C. A. Bertulani, and J. Ryckebusch, Quasi-free (p,2p) and (p,pn) reactions with unstable nuclei, Phys. Rev. C 88(6):064610 (2013).

[13] S. K. Charagi and S.K. Gupta, Coulomb-modified Glauber model description of heavy-ion reaction cross sections, Phys. Rev. C 41:1610-1618 (1990).

[14] V. Panin et al., Exclusive measurements of quasi-free proton scattering reactions in inverse and complete kinematics, Phys. Lett. B 753, 204-210 (2016).

[15] The separation energies are taken from the Nuclear data webpage http://www.nndc.bnl.gov/nudat2/.

[16] K. Blasche, The heavy ion synchrotron SIS, EPAC 92: Third European Particle Accelerator Conference, 9-13 (1992).

[17] H. Geissel et al., The GSI projectile fragment separator (FRS): a versatile magnetic system for relativistic heavy ions, Nucl. Instr. Meth. Phys. Research B 70286 (1992).

[18] S. Altstadt, ${ }^{13,14} \mathrm{~B}(\mathrm{n}, \mathrm{V})$ via Coulomb Dissociation to Constrain the Astrophysical r-Process, Dissertation, Johann Wolfgang Goethe-Universität, Germany, 2014.

[19] D. Bertini, R3BRoot, simulation and analysis framework for the R3B experiment at FAIR, J Phys. Conf. Ser. 331(3):032036 (2011).

[20] N.S. Chant and P.G. Roos, Distorted-wave impulse-approximation calculations for quasifree cluster knockout reactions, Phys. Rev. C 15, 57-68 (1977).

[21] C. Bertulani. private communication.

[22] L. Lapikas, Quasi-Elastic Electron Scattering off Nuclei, Nucl. Phys. A 553, 297c (1993).

[23] M. Leuschner et al., Quasielastic proton knockout from ${ }^{16}$ O, Phys. Rev. 187:1246-1258 (1969).

[24] M. Bernheim et al., The Influence of Bound State and Optical Potentials of 1p Momentum Distributions Obtained From ${ }^{12} \mathrm{C}$ and ${ }^{16} \mathrm{O}, \mathrm{P}(\mathrm{e}, \mathrm{e} \mathrm{p})$ Reactions, Phys. Rev. C 49:955-967 (1994).

[25] T. Aumann, Prospects of nuclear structure at the future GSI accelerators, PPNP 59, 3 (2007). 
